\title{
Human papillomavirus and herpes simplex virus infections in patients with mucocutaneous lesions can be linked to host TBX-21 gene polymorphism
}

\section{Amin Aziz Bakir*}

\section{Abstract}

Background and objective: Human papillomavirus (HPV) and herpes simplex-2 (HSV-2) are the common cause of genital lesions in women. The variation in host genetic makeup can determine the consequence of a viral infection in that host. T-bet gene polymorphism has been associated with the incidence of several types of infections. This study investigates the impact of $T$-bet polymorphism on the incidence of HPV and HSV in genital lesions.
\end{abstract}

Methods: 215 women, including 71 HPV infected, 72 HSV-2 infected, and 72 controls were enrolled. Viral genotyping was done on genital swab samples using Realtime PCR. In all participants, the extracted DNA from blood was tested for $T$-bet gene variation at Ch17.rs17244587 G>A site using ARMS-PCR. ELISA was done to participants sera to detect HSV-1 IgM.

Results: Genotyping of HPV infection revealed that $(73.0 \%)$ were at low risk. Most individuals $(72.5 \%)$ were homozygous GG, while $(20.9 \%)$ were heterozygous AG and $(6.5 \%)$ were homozygous AA, of which $92.8 \%$ were HSV-2 infected patients. None of 18 (8.4\%) HSV-1-IgM positive women were homozygous AA.

Conclusion: T-bet gene allele A appears to be linked with more incidence of HSV-2 in genital lesions, but such influence was not observed for HPV genotypes and HSV-1.

Keywords: HPV genotypes; HSV-2 infection; Genital lesion; T-bet polymorphism.

\section{Introduction}

Viruses are among the common causes of genital mucocutaneous infections. The predominant types of viral infections associated with genital lesions are human papillomavirus (HPV) and herpes simplex virus 2 (HSV-2) that are also recognized as globally prevalent sexually transmitted infections. ${ }^{1}$ Indeed, both HPV and HSV-2 have been reported to be associated with cervical cancer. ${ }^{2}$

HPV is a DNA virus that infects cutaneous and mucosal epithelia causing warts or benign lesions. ${ }^{3}$ However, the majority of infections resolve clinically after several weeks. ${ }^{4}$ HPV is a worldwide, sexually transmitted viral infection. More than 200 genotypes of HPV have been identified. Forty genotypes cause genital tract infections. ${ }^{5}$ According to carcinogenic properties, all HPV genotypes are divided into two groups. Firstly, high risk genotype group 16, 18, 31, 33, 35, 39, 45, 51, 52, $56,58,68$, and 59. Secondly, low-risk genotypes group, including53, 66, 70, 73, and 82. Each HPV genotype can be genetically differentiated based on transcriptional regulation factors, pathogenicity, geographical distribution, and immunological response. ${ }^{5}$ Persistent infection with the high-risk group is a major risk factor for dysplasia and cancer. ${ }^{6}$

HSV is a DNA virus that infects mucosal epithelial layers of the genitalia. ${ }^{7}$ Genital herpes is mainly caused by HSV-2 and less frequently by HSV-1. Both HSV types are structurally similar and about $74 \%$ identical at the genome level. ${ }^{8}$ Genital herpes infection is the most common sexually transmitted disease occurring in

${ }^{1}$ Department of Basic Sciences, College of Medicine, Hawler Medical University, Erbil, I raq.

* Correspondence: amin.bakir@hmu.edu.krd 
all age groups. ${ }^{9}$ The prognosis of infection can be presented with typical popular lesions that may progress to vesicles and ulcer formation. ${ }^{10}$ During the first infection with the HSV virus, clusters of CD4 and CD8 T lymphocytes are accumulated in the vaginal tissue. In the case of recurrent infection, CD8 T Cells are dominantly responsible for initiating the immune response. $^{8}$

Innate immunity, particularly interferon type-I response, is the first-line defense mechanism that is robust and effective against viral invasion. ${ }^{11}$ Variations in human genetics could form a variety of responses to viral infection. ${ }^{12}$ Indeed, such genetic variations and polymorphism in genes that control immune response have been part of human evolution against the viruses. ${ }^{13}$ One important gene with significant variations is TBX21 gene, which dramatically impacts the modulation of the immune system. ${ }^{14}$ It is responsible for switching the T-cell precursors towards IFN-Y producing cells like NK cells ${ }^{15}$ and CD8 T-cells. ${ }^{16}$ Polymorphism in TBX21 genes linked to a wide range of immunity mediated outcomes such as rheumatoid arthritis, ${ }^{17}$ asthma, ${ }^{18}$ autoimmune hepatitis, ${ }^{19}$, and susceptibility to viral infections. ${ }^{15}$ TBX21 gene is located on human chromosome 17 with several regions that have allelic variations, including rs17244587 G>A locus. ${ }^{20}$ Individuals with Ch17.rs17244587 homozygous AA have been found to be at greater risk of genital HSV-2 incidence. ${ }^{21}$

Given all these facts, it would be of great interest to find the link between the incidence of HPV and HSV with T-bet gene polymorphism in the Erbil women populace. Further, this study aimed to pursue the influence of TBX21 gene polymorphism on the incidence of HPV that causes similar disease and HSV-1 that shares similar genetics with HSV-2.

\section{Methods}

Study design and sampling:

A case-control study enrolled $71 \mathrm{HPV}$ and
72 HSV-2 infected females with genital warts referred to Genome Diagnosis Laboratory in Erbil, Iraq, from January to August 2020. Additionally, 72 age-matched female controls with no previous history of HPV and HSV-2 genital infections were included according to case-control criteria. ${ }^{22}$ Cervical swabs were collected only from HPV-infected and HSV-2Infected patients, while $5 \mathrm{~mL}$ venous blood was taken from every participant after verbal consent. The study has been approved by the Medical Research Ethics Committee of the College of Medicine, Hawler Medical University. Cervical swabs were taken by gynecologists from the genital region for viral DNA extraction and genotyping. Blood samples were mixed instantly after venipuncture at 20:1 v/v with $3 \%$ EDTA solution. $200 \mu \mathrm{L}$ of whole blood was used for human DNA extraction, and $50 \mu \mathrm{L}$ of plasma was taken for HSV-1 IgM detection.

\section{DNA extraction}

RealLine $^{\mathrm{TM}}$ Extraction 100 (Boiron, Jena, Germany) kit has been used to extract viral DNA from swab samples and genomic DNA from blood samples. In a $1.5 \mathrm{~mL}$ tube, $200 \mu \mathrm{L}$ of the cell suspension or whole blood was mixed with $30 \mu \mathrm{L}$ of internal control and $300 \mu \mathrm{L}$ of Lysis Reagent. $400 \mu \mathrm{L}$ of nucleic acid precipitation solution was added. After vortex and centrifuging at $13000 \mathrm{rpm}$ for $5 \mathrm{~min}$ at $25^{\circ} \mathrm{C}$, the supernatant was discarded, and the pellet was washed with $500 \mu \mathrm{L}$ wash solution twice. Pellet was dried then dissolved in $200 \mu \mathrm{L}$ nuclease-free water. Simultaneously, positive and negative controls per sample were set in separate tubes and treated exactly the same as the sample. The DNA solution was either directly used for PCR or stored at $-20^{\circ} \mathrm{C}$ for later use.

\section{Genotyping of viruses}

RealLine $^{\mathrm{TM}}$ HPV HCR Genotype and RealLine $^{\mathrm{TM}}$ HSV1/HSV2 kits (Boiron, Jena, Germany) were used for genotyping of HPV and HSV samples, respectively, as instructed by the manufacturer. Triplicate 
reactions were made per sample in addition to positive and negative control reactions, all of which contained internal control. The assay is based on real-time $\mathrm{PCR}$ run at $50^{\circ} \mathrm{C}$ for 2 min then polymerase activation at $95^{\circ} \mathrm{C}$ for 2 min followed by 50 cycles of $94^{\circ} \mathrm{C}$ for 10 seconds and $60^{\circ} \mathrm{C}$ for 20 seconds. The fluorescence was measured at $60^{\circ} \mathrm{C}$ for FAM, HEX, ROX, and Cy5 channels. The sample was considered positive for the corresponding genotype if the threshold was less than 35 . The HPV genotyping assay can detect HPV carcinogenic risk types: 16, 18, 31, 33, 35, 39, 45, 51, 52, 56, 58 AND 59. Genotype 16 and 18 are universally grouped as high risk, and the other ten genotypes are low-risk group. ${ }^{23}$ The genotyping of HSV was restricted to HSV-1 and HSV-2 types. Only HSV-2 types were included in this study.

\section{T-bet SNP typing:}

The isolated DNA from blood samples was tested for polymorphism in T-bet gene using ARMS-PCR method. Three primers were designed to detect single nucleotide polymorphism at Homo sapiens Ch17.rs17244587 G>A site. Two allele-specific forward primers: $1 \mu \mathrm{L}$ of dominant $\mathbf{G}$ allele primer of5'- GAAGACAAGAAAGTCTTGGG-3'and $1 \mu \mathrm{L}$ of the less common A allele primer:5'- GAAGACAAGAAAGTCTTGGA-3'each were added to a PCR tube. $1 \mu \mathrm{L}$ of common reverse primer 5'- CCTGTCGACCGGACCCGAGG -3 'was added to each tube in addition to $19 \mu \mathrm{L}$ PCR master mix containing dNTP, $\mathrm{MgCl}_{2}$, Taq-polymerase (Promega, USA), 100ng sample DNA, and nuclease free water to make a total volume of reaction at $20 \mu \mathrm{L}$. The PCR setting was started with 94C for 5 minutes, followed by 40 cycles of 94C (40 seconds), 60C (45 seconds), and $72 \mathrm{C}$ (60 seconds) that gives a product size of $241 \mathrm{bp}$ (Figure 1).

\section{Detection of serum HSV-1 IgM:}

The blood samples of participants were taken and investigated for the presence of HSV-1 IgM using DIA source Immuno-Assays SA kit (Louvain-la-Neuve,
Belgium). In a microtitration plate, $50 \mu \mathrm{L}$ of plasma was added to the solid phase anti-horse IgM antibody precoated well. After washing and discarding unbound antibodies and other plasma components, the HSV1 specific IgM captured on solid phase anti-horse IgM was detected by adding the inactivated labeled HSV-1 specific peroxidase conjugated antibodies. The reaction was incubated, followed by washing to remove unbound conjugate antibodies. Substrate (chromogen) was added that instantly hydrolyzes and generates color proportional to the amount of bound conjugate - IgM in the well. The microtitration plate was read at Optical Density of $450 \mathrm{~nm}$ wavelength and the reference filter of $630 \mathrm{~nm}$ in the ELISA plate reader. The calculation was done the cut-off formulation: Cut-Off $=N C+0.250$. A positive result indicates that the patient is undergoing an acute HSV-1 infection.

\section{Statistical analysis:}

Data analysis and graphing were done using the statistical package for the social sciences (SPSS version 20), GraphPad Prism v6, and R Studio v3.5.1 packages. Fisher exact test was applied to determine the association of different groups. A $P$ value of $\leq 0.05$ was considered statistically significant.

\section{Results}

Distribution of subjects according to age:

The three groups of participants were arranged based on age intervals. Fisher exact test was done to define the age variation among different groups (Table 1). No significant differences were detected between the three groups regarding the age distribution $(P=0.579)$.

\section{Genotyping of HPV samples:}

The number and percentage of low-risk and high-risk genotypes of HPV are depicted in Figure 1. 
Human papillomavirus and herpes simplex virus ...

Zanco J . Med. Sci., Vol. 25, No. (3), December, 2021

https:/ / doi.org/ 10.15218/ zjms.2021.033

Table 1 Correlation and age distribution between infected patients and control

\begin{tabular}{lcccc}
\hline Age intervals & $\begin{array}{c}\text { Controls } \\
\text { No (\%) }\end{array}$ & $\begin{array}{c}\text { HPV } \\
\text { No (\%) }\end{array}$ & $\begin{array}{c}\text { HSV-2 } \\
\text { No (\%) }\end{array}$ & $\begin{array}{c}\text { Total } \\
\text { No (\%) }\end{array}$ \\
\hline$\leq 20$ & $7(9.7)$ & $4(5.6)$ & $4(5.5)$ & $15(6.98)$ \\
$20-29$ & $23(31.9)$ & $32(45.1)$ & $36(50)$ & $91(42.3)$ \\
$30-39$ & $27(37.5)$ & $24(33.8)$ & $19(26.4)$ & $70(32.5)$ \\
$40-49$ & $11(15.7)$ & $7(9.8)$ & $10(13.9)$ & $28(13)$ \\
$\geq 50$ & $4(5.5)$ & $4(5.6)$ & $3(4.1)$ & $11(5.1)$ \\
Total & $72(100.0)$ & $71(100.0)$ & $72(100.0)$ & $215(100.0)$ \\
Fisher Exact test & & $P=0.579$ & \\
\hline
\end{tabular}

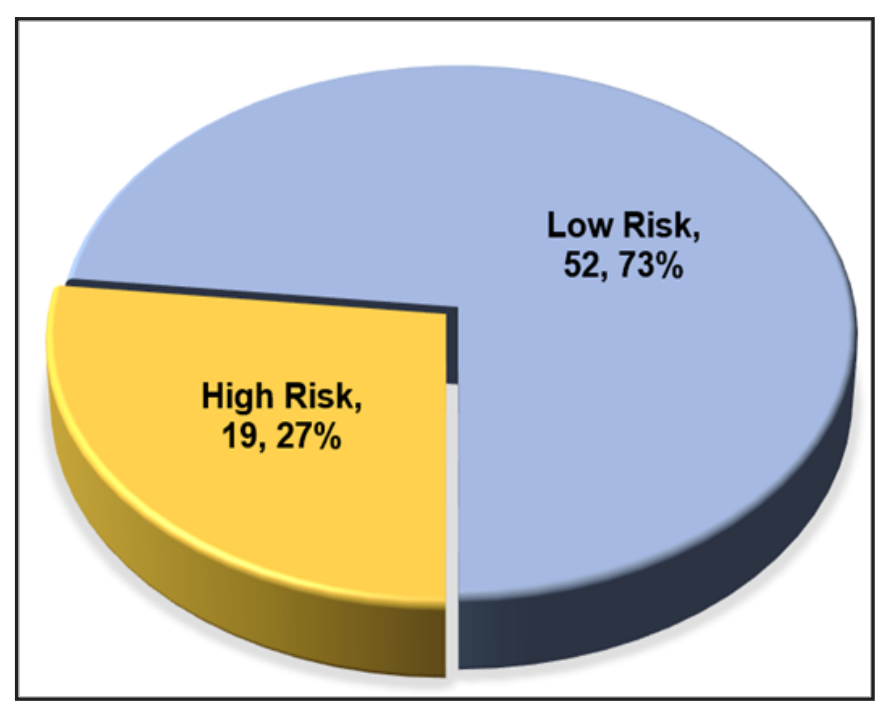

Figure 1 Distribution of genotype groups in HPV infected individuals 


\section{Gene typing of host TBX21}

The DNA isolated from blood samples of control and infected has been tested for polymorphism at Ch17.rs 17244587 G>A SNP using ARMS-PCR (Figure 2). The three genome types (i.e., homozygous GG, heterozygous $A G$, and homozygous $A A$ ) have been illustrated in (Table 2).
Significant differences in T-bet gene polymorphism were detected between HSV-2 and HPV $(P<0.001)$, and between HSV-2 and controls $(P<0.001)$. However, no significant differences were found between HPV infected individuals and Controls in their $T$-bet gene polymorphism.

Table 2 Frequency of T-bet genotypes at Ch17.rs17244587 in control and infected individuals

\begin{tabular}{lcccc}
\hline & GG & AG & AA & Total \\
\hline Control & $59(81.9 \%)$ & $12(16.7 \%)$ & $1(1.4 \%)$ & $72(100 \%)$ \\
HPV infected & $61(85.9 \%)$ & $10(14.1 \%)$ & $0(0.0 \%)$ & $71(100 \%)$ \\
HSV-2 infected & $36(50 \%)$ & $23(31.9 \%)$ & $13(18.1 \%)$ & $72(100 \%)$ \\
Total & $156(72.5 \%)$ & $45(20.9 \%)$ & $14(6.5 \%)$ & $215(100 \%)$ \\
Fisher Exact test & Control, HPV, HSV-2:P $<0.001$ & & \\
& Control vs. HPV: $P=0.546$ & & \\
& Control vs. HSV-2: $P<0.001$ & & \\
& HPC vs. HSV-2: $P<0.001$ & & \\
& & &
\end{tabular}

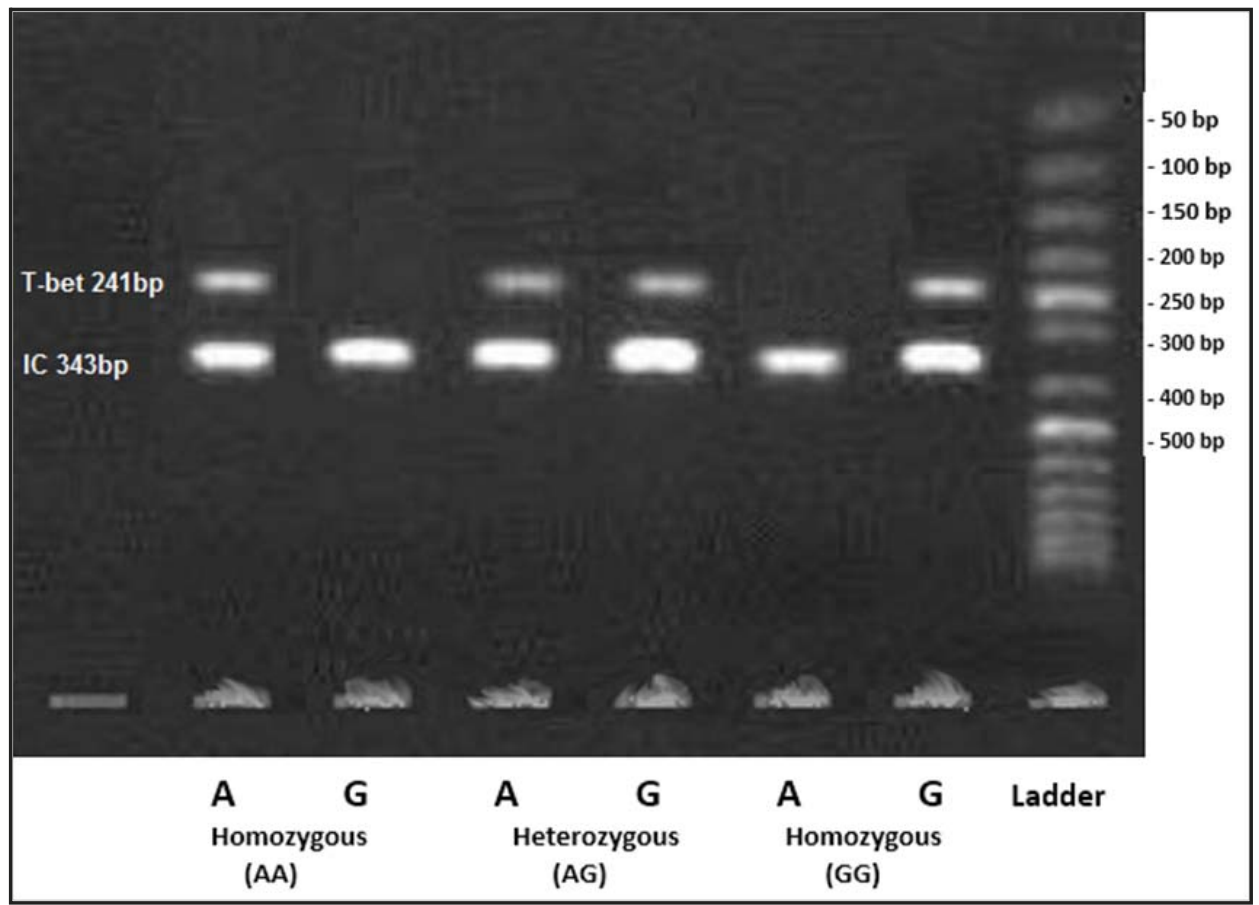

Figure 2 The isolated DNA from blood samples of control and infected patients for polymorphism at Ch17.rs 17244587 G>A SNP using ARMS-PCR 
T-bet polymorphism in patients infected with different HPV genotypes

The pattern of distribution of T-bet gene polymorphism (Ch17. Rs17244587) was investigated for its association with cervical cancer low-risk and high-risk groups in HPV infected patients (Figure 3). The statistical analysis showed the correlation between HPV genotypes and Host T-bet polymorphism distribution.
T-bet polymorphism according to HSV-1 IgM detection

The results of blood HSV-1 IgM tests of control and infected individuals were aligned with T-bet gene polymorphism (Table 3). No significant differences were found between HSV-1 IgM positive and negative groups $(P=0.413)$

Table 3 Distribution of T-bet alleles in HSV-1 IgM tested individuals

\begin{tabular}{lcccc}
\hline HSV-1 IgM & GG & AG & AA & Total \\
\hline Positive & $15(83.3 \%)$ & $3(16.7 \%)$ & $0(0.0 \%)$ & $18(100 \%)$ \\
Negative & $141(71.6 \%)$ & $42(21.3 \%)$ & $14(7.1 \%)$ & $197(100 \%)$ \\
Total & $156(72.5 \%)$ & $45(20.9 \%)$ & $14(6.5 \%)$ & $215(100 \%)$ \\
Fisher Exact test & & Control, HPV, HSV-2:P $=0.4139$ & \\
\hline
\end{tabular}

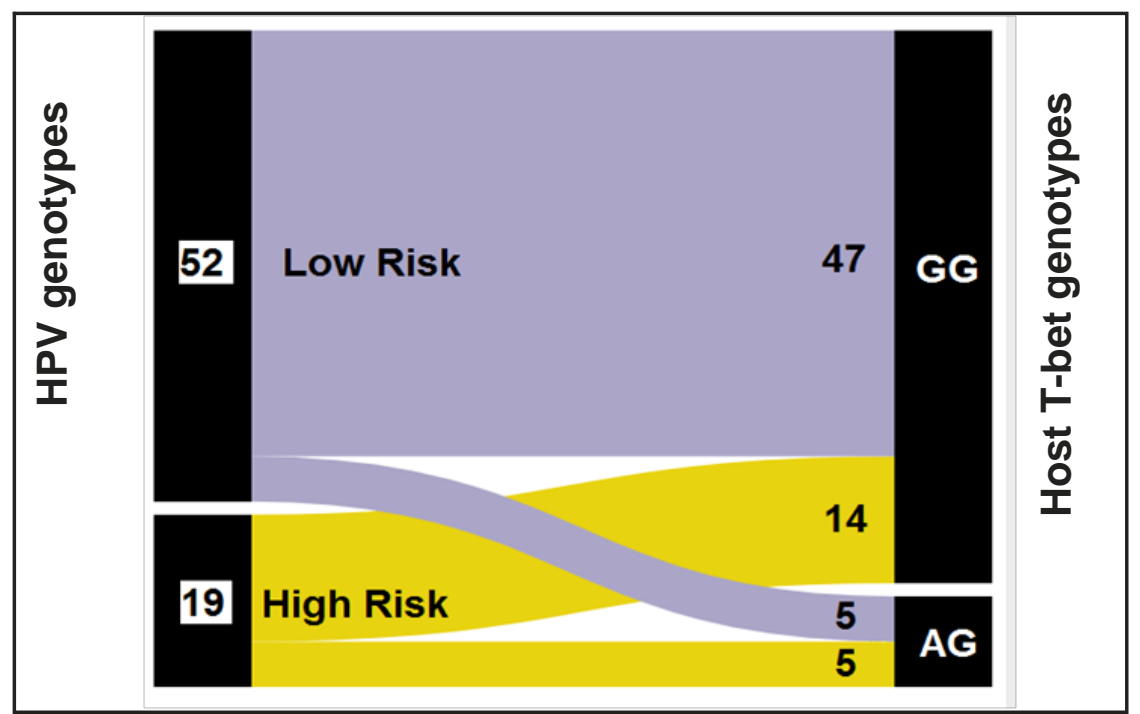

Figure 3 Parallel Set diagram of HPV cancer risk genotypes distribution according to the polymorphism of infected patients at Ch17. Rs17244587 G>A region of T-bet gene. Fisher Exact test: $P=0.1183$ 


\section{Discussion}

The susceptibility to infectious diseases is greatly influenced by human genes. It is likely that genes associated with the immune system play a key role in response to infectious diseases. ${ }^{12,24}$ Differences in severity and prevalence of pathogens have been revealed between and within the exposed populations. ${ }^{25}$ Among the groups taken in this study, the HSV-2 infected patients have been enrolled based on the role of the $T$-bet gene in genital infections. In view of that, $T$-bet gene has been found to be very important in the innate and acquired immune response against HSV-2 genital infections in mice ${ }^{26}$ and further documented in human genital infection. ${ }^{27}$ HPV infected patients were included due to the similarities in pathogenicity of HPV and HSV-2 infections for both inducing mucocutaneous genital infection. Indeed, HPV and HSV-2 are common sexually transmitted infections encountered in clinical practice and the most prevalent genital viral infections worldwide. ${ }^{28}$ Therefore, it is essential to investigate the extent of the same host genetic influence on both viruses. The number of case and control individuals enrolled in this study and their ages are randomized, considering the statistical representativeness of each group (Table 1).

Regarding the detection and genotyping of viruses among samples using Real-time PCR, HPV genotypes were further divided into two groups based on their potential risk in endorsing cervical cancer (Figure 1). The HPV samples were mainly low-risk genotypes $73 \%$, and the remaining $27 \%$ were high risk genotypes. These findings are comparable with the other epidemiological studies in the neighboring region. Thus, the high risk HPV were $32 \%$ among 6388 patients in the Turkish Gynecologic and Oncology Center, ${ }^{29}$ and $(28.5 \%)$ in infected Turkish women from the Manisa region. ${ }^{30}$

It is worth noting that there is no published data on the prevalence of HPV genotypes in the Kurdistan region of Iraq. Prior to including samples in the study, the vast majority $(97 \%)$ for the detection of HSV genotypes from pap smear were HSV-2 (data not shown). Therefore, HSV-1 was excluded from this study. Then all 215 included individuals in three studied groups were investigated for their T-bet polymorphism at Ch17. Rs17244587 using ARMS-PCR (Figure 2). ARMS-PCR has been widely used in research and is considered a less complicated alternative method for sequencing in studying point mutation and Single nucleotide polymorphism. ${ }^{31}$ The T-bet gene zygosity of a participant on Ch17. Rs 17244587 was targeted based on the findings of Svenson and coworker on the link between polymorphism at Rs7244587 in T-bet gene and incidence of HSV-2 genital infections in humans. $^{32}$ They found a significant association of polymorphism in this DNA sequence and HSV-2 infection, which is relatively in line with the finding of the current study (Table 2). Nevertheless, this genetic variation showed no statistical differences in infection with HPV compared to healthy controls. Thus, typing of Rs17244587 seems to be of great importance in HSV-2 infections. Perhaps determining the frequency of rs 17244587 SNPs in the population could provide an estimate of incidence for this type of viral infection. In view of that, the finding of this study can be a decent representation of ethnically Kurdish society in Erbil city. Overall, the homozygous GG genotype is predominant globally. ${ }^{33,34}$ Hence, the majority of individuals in this study were found to be homozygous GG followed by heterozygous GA and a small proportion of homozygous AA. The influence of $T$-bet gene heterozygosity on the incidence of different HPV risk genotypes was investigated. These findings suggested no differences between Rs17244587 homozygous GG and heterozygous GA in the incidence of HPV risk groups (Figure 3). Besides, there was a strong positive correlation between the frequency of genotypes and the distribution of 
T-bet gene alleles.

HSV-2 illustrates massive similarities with HSV-1 in replication, virulence, and drug activity. ${ }^{7}$ Since there is a significant influence of homozygous AA on HSV-2 incidence, it was interesting to pursue this influence on HSV-1 infections. To this end, all participants were tested for serum HSV-1 IgM. Nonetheless, both seropositive and seronegative groups showed no statistically significant differences in the distribution of their T-bet gene polymorphism (Table 3).

\section{Conclusion}

The findings of this study promote the possible link between host genetic makeup and the infectiveness of pathogens through such links that seems to be unique to certain viruses and limited to very specific loci on the human genome.

\section{Funding}

None.

\section{Competing interests}

None declared.

\section{References}

1. Torcia MG. Interplay among vaginal microbiome, immune response and sexually transmitted viral infections. Int J Mol Sci. 2019; 20(2):266. https://doi.org/10.3390/ijms20020266.

2. Bahena-Román $M$, Sánchez-Alemán $M A$, Contreras-Ochoa CO, Lagunas-Martínez A, Olamendi-Portugal M, López-Estrada G, et al. Prevalence of active infection by herpes simplex virus type 2 in patients with high-risk human papillomavirus infection: A cross-sectional study. J Med Virol. 2020; 92(8):1246-52. https://doi.org/10.1002/jmv.25668.

3. Graham SV. The human papillomavirus replication cycle, and its links to cancer progression: a comprehensive review. Clin Sci. 2017; 131(17):2201-21. https://doi.org/110.1042/ CS20160786.

4. Norenhag J, Du J, Olovsson M, Verstraelen H, Engstrand L, Brusselaers $\mathrm{N}$. The vaginal microbiota, human papillomavirus and cervical dysplasia: a systematic review and network meta-analysis. BJOG An Int J Obstet Gynaecol. 2020; 127(2):171-80. https://doi.org/10.1111/1471 -0528.15854 .

5. Chan CK, Aimagambetova G, Ukybassova T, Kongrtay K, Azizan A. Human papillomavirus infection and cervical cancer: Epidemiology, screening, and vaccination - Review of current perspectives. J Oncol. 2019; 2019:3257939. https://doi.org/10.1155/2019/3257939.

6. Lin C, Franceschi S, Clifford GM. Human papillomavirus types from infection to cancer in the anus, according to sex and HIV status: a systematic review and meta-analysis. Lancet Infect Dis. 2018; 18(2):198-206. https://doi.org/10.1016/S1473-3099(17)30653-9.

7. Koelle DM, Norberg P, Fitzgibbon MP, Russell RM, Grninger AL, Huang ML, et al. Worldwide circulation of HSV-2 $\times$ HSV-1 recombinant strains. Sci Rep. 2017; 7(February): 1-12. https://doi.org/10.1038/srep44084.

8. Kim HC, Lee HK. Vaccines against genital herpes: Where are we? Vaccines. 2020; 8(3): 1-13. https://doi.org/10.3390/vaccines8030420.

9. Simonetta F, Pradier A, Roosnek E. T-bet and eomesodermin in NK cell development, maturation, and function. Front Immunol. 2016; 7:241. https://doi.org/10.3389/fimmu.2016.00241.

10. Patel R, Kennedy OJ, Clarke E, Cowan FM, Kinghorn GR, Munday PE, et al. European guidelines for the management of genital herpes. Int J STD AIDS. 2017; 28(14):1366-79. https://doi.org/10.1177/0956462417727194.

11. Huang Y, Dai H, Ke R. Principles of Effective and Robust Innate Immune Response to Viral Infections: A Multiplex Network Analysis. Front Immunol. 2019; 10(July):1736. https://doi.org/10.3389/fimmu.2019.01736.

12. Kenney AD, Dowdle JA, Bozzacco L, McMichael TM, St. Gelais C, Panfil AR, et al. HHS Public Access. 2018: 51:241-63. https://doi.org/10.1146/annurev-genet-120116023425.

13. McLaughlin RN, Malik HS. Genetic conflicts: The usual suspects and beyond. J Exp Biol. 2017; 220(1):6-17. https://doi.org/10.1242/jeb.148148.

14. Casaca VI, Illi S, Suttner K, Schlpeich I, Ballenberger N, Klucker $\mathrm{E}$, et al. TBX21 and HLX1 polymorphisms influence cytokine secretion at birth. PLoS One. 2012; 7(1):1-8. https://doi.org/10.1371/journal.pone.0031069.

15. Zhu DY, Deng XZ, Zhu YM, Li GT, Zhang GQ, Wang LJ, et al. Association of IL-28B, TBX21 gene polymorphisms and predictors of virological response for chronic hepatitis C. Arch Virol. 2018; 163(5):1253-62. https://doi.org/10.1007/s00705018-3750-9.

16. Sivori S, Pende D, Quatrini L, Pietra G, Chiesa MD, Vacca $P$, et al. NK cells and ILCs in tumor immunotherapy. Mol Aspects Med. 2020; 80:100870. https://doi.org/10.1016/ j.mam.2020.100870.

17. Ge M, Zheng Y, Li X, Shi J, Ge J. The polymorphisms of $T$ cell-specific TBX21 and STAT4 genes may contribute to the susceptibility of Chinese individuals to aplastic anemia. Hum Immunol. 2012; 73(1):118-21. https://doi.org/10.1016/j.humimm.2011.11.007. 
18. White AA, Stevenson DD. Aspirin-exacerbated respiratory disease. N Engl J Med. 2018; 379(11):1060-70. https://doi.org/10.1056/ NEJMra1712125.

19. Sun W, Wu HY, Chen S. Influence of TBX21 T-1993C variant on autoimmune hepatitis development by Yin-Yang 1 binding. World J Gastroenterol. 2017; 23(48):8500-11. https://doi.org/10.3748/wjg.v23.i48.8500.

20. Lazarevic V, Glimcher LH. T-bet in disease. Nat Immunol. 2011; 12(7):597-606. https://doi.org/10.1038/ni.2059.

21. Sánchez D, Lefebvre C, García LF, Barrera LF. Variants in the IFNy transcription factor genes TBET, STAT1, STAT4, and HLX and the risk of pulmonary tuberculosis in a Colombian population: a case-control study. Biomedica. 2013; 33(2):259-67.

22. Mansournia MA, Jewell NP, Greenland S. Case-control matching: effects, misconceptions, and recommendations. Eur J Epidemiol. 2018; 33(1):5-14. https://doi.org/10.1007/s10654-0170325-0.

23. Mahmoodi $P$, Fani $M$, Rezayi $M$, et al. Early detection of cervical cancer based on high-risk HPV DNA-based genosensors: A systematic review. Bio Factors. 2019; 45(2):101-17. https://doi.org/10.1002/biof.1465.

24. Xavier-Carvalho C, Cardoso CC, de Souza Kehdy F, Pacheco AG, Moraes MO. Host genetics and dengue fever. Infect Genet Evol. 2017; 56:99-110. https://doi.org/10.1016/ j.meegid.2017.11.009

25. Chapman SJ, Hill AVS. Human genetic susceptibility to infectious disease. Nat Rev Genet. 2012; 13(3):175-88. https://doi.org/ 10.1038/nrg3114.

26. Zhang Y, Zhang Y, Gu W, He L, Sun B. Th1/Th2 cell's function in immune system. In: $T$ helper cell differentiation and their function. Springer 2014; 45-65. https://doi.org/10.1007/978-94-017-9487 $-93$.

27. Svensson A, Tunbäck P, Nordström I, Padyukov $\mathrm{L}$, Eriksson K. Polymorphisms in Toll-like receptor 3 confer natural resistance to human herpes simplex virus type 2 infection. J Gen Virol. 2012; 93(8):1717-24. https://doi.org/10.1099/ vir.0.042572-0.

28. Viera $\mathrm{MH}, \mathrm{Amini} \mathrm{S}$, Huo R, Konda S, Block S, Berman B. Herpes simplex virus and human papillomavirus genital infections: new and investigational therapeutic options. Int J Dermatol. 2010; 49(7):733-49. https://doi.org/10.1111/j.1365 $-4632.2009 .04375 . x$.

29. Dursun P, Ayhan A, Mutlu L, et al. HPV Types in Turkey: Multicenter hospital based evaluation of 6388 patients in Turkish gynecologic oncology group centers. Turkish J Pathol. 2013; 29(3): 210-6. https://doi.org/10.5146/tjpath.2013.01188.

30. Akcali S, Goker A, Ecemis T, Kandiloglu AR, Sanlidag T. Human papilloma virus frequency and genotype distribution in a Turkish population. Asian Pacific J Cancer Prev. 2013; 14(1):503-6. https://doi.org/10.7314/apjcp.2013.14.1.503.

31. Cossu G, Previtali SC, Napolitano S, Cicalese MP, Tedesco FS, Nicastro F, et al. Intra-arterial transplantation of HLA-matched donor mesoangioblasts in Duchenne muscular dystrophy. EMBO Mol Med. 2015; 7(12):1513-28. https://doi.org/10.15252/emmm.201505636.

32. Rubicz R, Leach CT, Kraig E, Dhurndhar N, Duggirala R, Blangero J, et al. Genetic factors influence serological measures of common infections. Hum Hered. 2011; 72(2):133-41. https://doi.org/10.1159/000331220.

33. Bakkeheim E, Mowinckel P, Carlsen KH, Håland G, Carlsen KCL. Paracetamol in early infancy: the risk of childhood allergy and asthma. Acta Paediatr. 2011; 100(1):90-6. https://doi.org/ 10.1111/j.1651-2227.2010.01942.x.

34. Rodriguez-Flores JL, Fakhro K, Agosto-Perez F, Ramstetter MD, Arbiza L, Vinncent TL, et al. Indigenous Arabs are descendants of the earliest split from ancient Eurasian populations. Genome Res. 2016; 26(2):151-62. https://doi.org/10.1101/ gr.191478.115. 